\title{
The curious case of thyroid dysfunction and the monoclonal antibody
}

Faisal Hasan ${ }^{1}$, Irfan Khan ${ }^{1}$ A.P. Lambert ${ }^{1}$

${ }^{1}$ Department of Diabetes and Endocrinology, Musgrove Park Hospital, Taunton and Somerset Foundation Trust, Taunton, Somerset

\section{INTRODUCTION}

- Alemtuzumab was the first humanised monoclonal antibody. It is used in haematological malignancies and multiple sclerosis.

- It is associated with secondary autoimmune adverse effects including Graves' disease, hypothyroidism, Goodpasture's disease and ITP.

\section{THE CASE}

A 46-year old male

Background: Relapsing remitting multiple sclerosis July 2011.

2 relapses on $\beta$-IFN-1a.

Rx: Alemtuzumab infusion $12 \mathrm{mg}$ for 5 days (12/08/12)

12 months later $12 \mathrm{mg}$ for 3 days (12/08/13)

No family history of autoimmune disease

No dysthyroid symptoms

Deranged thyroid tests on regular monitoring 25 months after last infusion

Examination: Pulse 68/min, regular

No goitre or neck nodules

No peripheral or eye signs of Graves' disease

\begin{tabular}{l|c|c|c|}
\hline Date \& Drug Treatment & $\begin{array}{c}\text { TSH (mu/l, } \\
0.34-5.6)\end{array}$ & $\begin{array}{c}\text { FT3 (pmol/l, } \\
3.8-6.0)\end{array}$ & $\begin{array}{c}\text { FT4 (pmol/l, } \\
7.9-20.0)\end{array}$ \\
\hline $26 / 03 / 15$ & 2.69 & & \\
\hline $28 / 07 / 15$ & 0.93 & & 54.0 \\
\hline $\begin{array}{l}\text { Carbimazole } 20 \mathrm{mg} / \mathrm{d} \\
\text { 28/09/15 } \\
\text { Carbimazole 5 mg/d }\end{array}$ & 0.18 & 28.9 & 10 \\
\hline $\begin{array}{l}\text { 03/11/2015 } \\
\text { Levothyroxine 50 mcg/d }\end{array}$ & 75.8 & 3.6 & 4.6 \\
\hline 24/12/2015 & 7.5 & 5.0 & 12.5 \\
\hline
\end{tabular}

\section{Other Investigations:}

-Anti TPO antibodies- positive at $65 \mathrm{IU} / \mathrm{ml}(0-8)$

-Anti TSH Receptor antibodies positive at $>40 \mathrm{U} / \mathrm{l}(<1.8)$

Levothyroxine was increased to $100 \mathrm{mcg} / \mathrm{d}$, patient now doing well.

\begin{tabular}{|l|c|c|c|c|}
\hline Adverse effects & \multicolumn{4}{|c|}{ Care MS I \& II Trials 1 } \\
& $\begin{array}{c}\text { Year 1 } \\
(\mathrm{n}=811)\end{array}$ & $\begin{array}{c}\text { Year 2 } \\
(\mathrm{n}=810)\end{array}$ & $\begin{array}{c}\text { Year 3 } \\
(\mathrm{n}=772)\end{array}$ & $\begin{array}{c}\text { Year 4 } \\
(\mathrm{n}=731)\end{array}$ \\
\hline Hyperthyroidism & $1.2 \%$ & $4.1 \%$ & $11.9 \%$ & $6.0 \%$ \\
\hline Hypothyroidism & $1.7 \%$ & $2.7 \%$ & $4.9 \%$ & $2.7 \%$ \\
\hline Thyroiditis & $0.7 \%$ & $1.5 \%$ & $1.9 \%$ & $1.4 \%$ \\
\hline
\end{tabular}

\section{DISCUSSION}

1. The incidence of thyroid dysfunction associated with Alemztuzumab can be up to $36 \%{ }^{2}$.

2. Mechanism of autoimmunity: profound lymphopenia and raised IL-21 levels increase cell cycling, leading to self reactive T-cells.

3. A range of thyroid disorders can be associated with Alemtuzumab - Graves' disease, thyroiditis, subclinical thyrotoxicosis and hypothyroidism ${ }^{3}$.

4. Patients can have no symptoms, as in our case, so regular monitoring is essential.

5. Anti-thyroid drugs, radioiodine treatment and surgery have all been used successfully in this condition ${ }^{4}$.

\section{Take Home Messages}

- Alemtuzumab causes autoimmune thyroid dysfunction in about $30 \%$ cases

- Regular monitoring of thyroid tests following Alemtuzumab are required for at least 4 years after the last infusion

\section{REFERENCES}

1. Twyman C, Oyuela P, Palmer J, Margolin D, Dayan C. Thyroid autoimmune adverse events in patients treated with alemtuzumab for relapsing-remitting multiple sclerosis: Four-year follow-up of the CARE-MS studies (P2.199). Neurology. 2014;82

2. Mahzari M, Arnaout A, Freedman MS. Alemtuzumab Induced Thyroid Disease in Multiple Sclerosis: A Review and Approach to Management. Can J Neurol Sci. 2015 Sep;42(5):284-91.

3. Daniels GH, Vladic A, Brinar V, et al. Alemtuzumab-related thyroid dysfunction in a phase 2 trial of patients with relapsing-remitting multiple sclerosis. J Clin Endocrino Metabol. 2014;99:80-9

4. Daniels GH, Vladic A, Brinar V, et al. Alemtuzumab-related thyroid dysfunction in a phase 2 trial of patients with relapsing-remitting multiple sclerosis. J Clin Endocrinol Metabol. 2014;99:80-9 